\title{
ON THE SECOND DUAL OF THE SPACE OF CONTINUOUS FUNCTIONS
}

\author{
BY \\ SAMUEL KAPLAN
}

Introduction. By "the space of continuous functions" we mean the Banach space $C$ of real continuous functions on a compact Hausdorff space $X$. $C$, its first dual, and its second dual are not only Banach spaces, but also vector lattices, and this aspect of them plays a central role in our treatment.

In $\$ \S 1-3$, we collect the properties of vector lattices which we need in the paper. In $\S 4$ we add some properties of the first dual of $C$ - the space of Radon measures on $X$-denoted by $L$ in the present paper. In $\S 5$ we imbed $C$ in its second dual, denoted by $M$, and then identify the space of all bounded real functions on $X$ with a quotient space of $M$, in fact with a topological direct summand. Thus each bounded function on $X$ represents an entire class of elements of $M$.

The value of an element $f$ of $M$ on an element $\mu$ of $L$ is denoted as usual by $f(\mu)$. If $f$ lies in $C$ then of course $f(\mu)=\mu(f)$ (usually written $\int f d \mu$ ), and thus the multiplication between $M$ and $L$ is an extension of the multiplication between $L$ and $C$. Now in integration theory, for each $\mu \in L$, there is a standard "extension" of $\int f d \mu$ to certain of the bounded functions on $X$ (we confine ourselves to bounded functions in this paper), which are consequently called $\mu$-integrable. The question arises: how is this "extension" related to the multiplication between $M$ and $L$. $\S \S 6-8$ (and $\S 12$ ) are devoted to this question. We show that given a bounded function $f$ on $X$ the equivalence class of $M$ represented by $f$ contains two distinguished elements, which we denote by $f_{*}$ and $f^{*}$, such that for every Radon measure $\mu, f_{*}(\mu)=\int_{*} f d \mu$ and $f^{*}(\mu)=\int^{*} f d \mu$, where $\int_{*}$ and $\int^{*}$ are the lower and upper integrals of $f$ with respect to $\mu$. Moreover, if $f$ is integrable for every Radon measure, then $f_{*}$ and $f^{*}$ coincide, and we have a unique element whose value on each $\mu$ is $\int f d \mu$.

In $\S 9$ we study order-convergence in $M$. It is in this area that $M$ offers its most striking immediate gain in clarity over consideration of only the bounded functions on $X$. We cite two examples. The first example: The characteristic functions of finite subsets of the real interval $0 \leqq x \leqq 1$ form a directed system which order-converges to the constant function 1 , while their Lebesgue integrals, being all zero, do not converge to the Lebesgue integral of 1 . In $M$, however, this directed system order-converges to an element different from 1 , and the above unsatisfactory situation disappears. In point of fact, order-convergence is perfectly well-behaved in $M:$ if a directed system order converges, its values on every Radon measure converge

Presented to the Society, October 27, 1956; received by the editors July 16, 1956. 
to the value of the limit element. The second example: Every bounded function on $X$ is the limit under order-convergence of some directed system of continuous functions; thus, unlike sequences, general directed systems seem to be useless for distinguishing "nice" functions (e.g. Borel) from completely arbitrary ones. If we turn to $M$, however, the elements which are limits under order-convergence of directed systems of continuous functions are precisely the unique elements discussed at the end of the last paragraph above.

In $\$ 11$ we examine a natural locally convex topology on $M$, first considered, to our knowledge, by Dieudonné $[4 ; 5]$. It is essentially the coarsest topology on $M$ which is related to the lattice properties of $M$ [13]. $M$ is complete under this topology [4]. Our principal result is that under the topology $C$ is dense in $M$.

$\$ 12$ makes a beginning on the detailed relationship between $M$ and integration theory as it is usually carried out on the bounded functions on $X$.

Part of the work on this paper was done during the summer of 1955, while the author was a guest of the Mathematics Department at the University of Chicago.

1. Ideals. We assume a knowledge of the basic definitions and elementary properties of vector lattices $[1 ; 2]$. As we have stated in the introduction, in this section and the following two sections we collect, without proofs, the standard properties of vector lattices which we will require.

We will call a linear subspace $I$ of a vector lattice $E$ an $i d e a l$ if it satisfies the condition

$$
a \in I,|b| \leqq|a| \text { implies } b \in I .
$$

It follows immediately that for every $a \in I,|a|, a^{+}, a^{-}$are all in $I$; hence that for every $a \in I, b \in I$, we have $a \bigvee b \in I, a \wedge b \in I$. Thus $I$ is itself a vector lattice. We also have

(1.2) Given a collection of ideals in a vector lattice, the linear subspace generated by their set-union is again an ideal.

A linear subspace $F$ of a vector lattice $E$ will be called closed if for every subset $A$ of $F, b=\bigvee A$ or $b=\bigwedge A$ implies $b \in F$; it will be called $\sigma$-closed if the condition is satisfied for every countable subset of $F$. For an ideal $I$ to be closed it is sufficient that for every subset $A$ of $I_{+}$(the positive cone of $I$ ), $b=\mathrm{V} A$ implies $b \in I$; and similarly for $\sigma$-closed.

We will call two elements $a, b$ of a vector lattice $E$ disjoint if $|a| \wedge|b|=0$. Given a set $A$ in $E$, the set of elements of $E$ each disjoint from all elements of $A$ is denoted by $A^{\prime}$. We have

(1.3) For any set $A$ in a vector lattice $E, A^{\prime}$ is a closed ideal.

(1.4) Let a vector lattice $E$ be the direct sum of two ideals $I_{1}, I_{2}: I_{1}+I_{2}=E$, $I_{1} \cap I_{2}=0$. For each $a \in E$ denote the component of $a$ in $I_{i}$ by $a_{i}(i=1,2)$. Then:

$\left(1^{\circ}\right)$ For every $a \in E, b \in E, a \leqq b$ if and only if $a_{i} \leqq b_{i}, i=1,2$;

$\left(2^{\circ}\right)$ If $b=\bigvee A$, then $b_{i}=\bigvee A_{i}$, where $A_{i}=\left\{a_{i} \mid a \in A\right\}(i=1,2)$; 
$\left(3^{\circ}\right)$ For each $a \geqq 0, a_{i}=\bigvee_{b \in I, b \S a} b(i=1,2)$;

(4. $I_{2}=\left(I_{1}\right)^{\prime}, I_{1}=\left(I_{2}\right)^{\prime}$.

Corollary. Under the above hypotheses, for every $a \in E, b \in E$, we have $(a \bigvee b)_{i}=a_{i} \bigvee b_{i}$ and $(a \wedge b)_{i}=a_{i} \wedge b_{i}(i=1,2)$. In particular, $\left(a^{+}\right)_{i}=\left(a_{i}\right)^{+}$, $\left(a^{-}\right)_{i}=\left(a_{i}\right)^{-},|a|_{i}=\left|a_{i}\right|$.

A subset $A$ of a vector lattice $E$ is said to be bounded above (below) if there exists $b \in E$ such that $a \leqq b(a \geqq b)$ for every $a \in A$. If it is bounded above and below, it is simply called bounded. Alternatively, $A$ is bounded if there exists $b \geqq 0$ such that $|a| \leqq b$ for every $a \in A$. $E$ will be called complete if for every set $A$ bounded above, $\mathrm{V} A$ exists in $E$ (and hence for every set $A$ bounded below, $\Lambda A$ exists in $E$ ).

Given a subset $A$ of a vector lattice $E$, the intersection of all ideals (closed ideals) containing $A$ is called the ideal (closed ideal) generated by $A$.

(1.5) (The Riesz TheOREM). Let $E$ be a complete vector lattice. Then if $A$ is any subset of $E$,

$\left(1^{\circ}\right)\left(A^{\prime}\right)^{\prime}$ is the closed ideal generated by $A$;

$\left(2^{\circ}\right) E$ is the direct sum of $A^{\prime}$ and $\left(A^{\prime}\right)^{\prime}$.

A proof of this theorem can be found in [2, Chapter II, $\$ 1$, Theorème 1].

2. The bounded linear functionals. Given a vector lattice $E$, a partial order is defined in the space of linear functionals on $E$ as follows: For two such linear functionals $\phi, \psi$, we have $\phi \leqq \psi$ if $\phi(a) \leqq \psi(a)$ for all $a \in E_{+}$. In particular $\phi \geqq 0$ (the zero functional) if $\phi(a) \geqq 0$ for all $a \in E_{+}$; such a linear functional is called positive.

A linear functional $\phi$ will be called bounded if $\sup _{a \in A}|\phi(a)|<\infty$ for every bounded set $A$. Under the above partial order, the set $\Omega(E)$ (or simply $\Omega$ ) of bounded linear functionals forms a complete vector lattice whose positive cone is the set of positive linear functionals [2, pp. 34-36]. The following two theorems give the lattice properties of $\Omega$ explicitly.

(2.1) Given $\phi \in \Omega, \psi \in \Omega$, then for every $a \in E_{+}$,

$$
(\phi \vee \psi)(a)=\sup _{b \in E_{+}, c \in E_{+}, b+c=a}[\phi(b)+\psi(c)]
$$

In particular,

$$
\phi^{+}(a)=\sup _{0 \leqq b \leqq a} \phi(b) \quad \text { and } \quad|\phi|(a)=\sup _{|b| \leqq a} \phi(b) .
$$

This last in turn gives us that for any $a \in E,|\phi(a)| \leqq|\phi|(|a|)$.

We will say a set $A$ in a vector lattice is directed under $\leqq(\geqq)$ if for every $a \in A, b \in A$, there exists $c \in A$ such that $a \leqq c, b \leqq c(a \geqq c, b \geqq c)$.

(2.2) Let $A$ be a subset of $\Omega$ and $\phi=\bigvee A$. Then for every $a \in E_{+}, \phi(a)$ $\geqq \sup _{\psi \in A} \psi(a)$. If in addition $A$ is directed under $\leqq$, then $\phi(a)=\sup _{\psi \in A} \psi(a)$. Similar statements hold for $\bigwedge A$. 
We will also need the following.

(2.3) Let $E$ be a complete vector lattice and I a closed ideal in E. Then $I^{\perp}$ and $\left(I^{\prime}\right)^{\perp}$ are closed ideals in $\Omega$ and $\left(I^{\prime}\right)^{\perp}=\left(I^{\perp}\right)^{\prime}$.

( $I^{\perp}$ is the set of elements in $\Omega$ which have value zero on all elements of $I$ ).

3. Banach lattices. A Banach lattice $E$ is a linear space which is both a Banach space and a vector lattice, and in which the norm and order satisfy the relation

$$
|a| \leqq|b| \text { implies }\|a\| \leqq\|b\| \text {. }
$$

In particular $\|a\|=\||a|\|$ for every $a \in E$.

Since $|a \vee c-b \vee c| \leqq|a-b|$ and $|a \wedge c-b \wedge c| \leqq|a-b|[1$, p. 220], the above gives us

(3.2) In a Banach lattice, $\|a \vee c-b \vee c\| \leqq\|a-b\|$ and $\|a \wedge c-b \wedge c\|$ $\leqq\|a-b\|$. Thus the operations $\vee$ and $\wedge$ are uniformly continuous under the norm.

This continuity gives us in turn

(3.3) $E_{+}$is closed under the norm.

(3.4) If an upper (lower) bound $b$ of a set $A$ is a limit point of $A$ under the norm, then $b=\bigvee A(b=\Lambda A)$.

(3.5) A closed ideal of $E$ is closed under the norm.

(3.6) The closure under the norm of an ideal is again an ideal.

(3.7) If $E$ is the direct sum of two ideals, then it is their topological direct sum.

Finally (via [1, p. 248] and (2.1))

(3.8) If $E$ is a Banach lattice, $\Omega$ coincides with the Banach space dual of $E$, and under this dual space norm, is itself a Banach lattice.

Henceforth, by the dual of a Banach lattice $E$, we will mean the above Banach lattice.

4. The first dual, $L$. Throughout this paper $X$ will be a fixed compact Hausdorff space. We denote by $C$ the space of continuous real functions on $X$ with norm defined by $\|f\|=\sup _{x \in X}|f(x)|$ and order defined by $f \leqq g$ if $f(x)$ $\leqq g(x)$ for all $x \in X$. Under these definitions $C$ is a Banach lattice. In fact it is an $(M)$-space with a strong order unit [11]: $f \geqq 0, q \geqq 0$ implies $\|f \vee g\|$ $=\max (\|f\|,\|g\|)$, and $\|f\| \leqq 1$ if and only if $|f| \leqq 1$ (the constant function everywhere equal to 1 ).

We denote the dual of $C$ by $L$. It is commonly called the space of Radon measures on $X$. The existence of 1 in $C$ gives immediately.

(4.1) For every $\mu \in L,\|\mu\|=|\mu|(1)$.

This in turn gives that $L$ is an (L)-space [10]: $\mu \in L_{+}, \nu \in L_{+}$implies $\|\mu+\nu\|=\|\mu\|+\|\nu\|$. As our interest in the present paper is in the dual of $L$, we give here only those properties of $L$ which we will require. The following is a converse of (3.4); it is not true in a general Banach lattice ( $C$, for example). 
(4.2) If a set $A$ in $L$ is directed under $\leqq$ and $\mathrm{V} A=\mu$, then $\mu$ is a limit point of $A$ under the norm.

Proof. From (2.2), $\mu(1)=\sup _{\nu \in A} \nu(1)$. Writing this in the form $\inf _{\nu \in A}(\mu-\nu)(1)=0$ and using the identity $(\mu-\nu)(1)=\|\mu-\nu\|$, we have the required conclusion.

(4.3) An ideal $I$ in $L$ is closed if and only if it is closed under the norm.

Proof. As we have remarked (3.5), the "only if" holds in any Banach lattice. Now suppose $I$ is closed under the norm, and consider a subset $A$ of $I_{+}$ with $b=\mathrm{V} A$. Let $B$ be the set obtained from $A$ by including the suprema of all finite subsets of $A$. Then $B C I_{+}, B$ is directed under $\leqq$, and $b=\mathrm{V} B$. It follows from (4.2) that $b$ is a limit point of $B$, hence of $I$.

While it is not in general true that a subset of $L$ which is bounded in the norm is bounded, we have

(4.4) If a subset $A$ of $L$ is directed under $\leqq$ and bounded in the norm, then $\checkmark A$ exists.

A proof of this, or rather the more general theorem (11.6) is given in [5, Théorème 1].

We will consider the space $X$ as a subset of $L$ by identifying each $x \in X$ with the element of $L$ defined by $x(f)=f(x)$ for all $f \in C$. It is immediate that for each $x \in X, x>0$ and $\|x\|=1$. We will denote by $L_{0}$ the linear subspace of $L$, closed under the norm, which is generated by $X$.

(4.5) $L_{0}$ is a closed ideal in $L$.

Proof. From (4.3) we need only show $L_{0}$ is an ideal; (3.6) says it is sufficient to show this for the linear subspace generated by $X$; and finally (1.2) says we can confine our attention to the one-dimensional linear subspace generated by a single $x \in X$. Suppose $0 \leqq \mu \leqq \lambda x$, where $\lambda$ is a positive real number. We remark first that for any two elements $f, g$ of $C, f(x)=g(x)$ implies $\mu(f)=\mu(g):$ for $|\mu(f-g)| \leqq \mu(|f-g|) \leqq(\lambda x)(|f-g|)=\lambda[x(|f-g|)]$ $=\lambda[|f-g|(x)]=0$. Then for any $f \in C_{+}, \mu(f)=f(x) \mu[f / f(x)]=f(x) \mu(1)$ $=\mu(1) x(f)$. Thus $\mu=\mu(1) x$. The argument is now easily carried through for a general $\mu$ such that $|\mu| \leqq x$.

We will denote $\left(L_{0}\right)^{\prime}$ in $L$ by $L_{1}$. Then (3.7) $L$ is the topological direct sum of $L_{0}$ and $L_{1} . L_{0}$ is the set of purely atomic (Radon) measures on $X$ and $L_{1}$ is the set of nonatomic ones. There is another characterization of $L_{0}$. Consider $\mu=\sum_{1}^{n} \lambda_{i} x_{i}$ (the $\lambda_{i}$ 's real numbers). We can clearly think of $\mu$ as a real function on $x$ with $\mu\left(x_{i}\right)=\lambda_{i}(i=1, \cdots, n)$ and $\mu(x)=0$ for all other $x \in X$, and with $\|\mu\|=\sum_{1}^{n}\left|\lambda_{i}\right|$. Since $L_{0}$ is the completion under this norm of the space of all such functions, we have

(4.6) $L_{0}$ can be identified with $l^{1}$ on $X$, that is, the Banach lattice of all real functions $\mu$ on $X$ satisfying $\sum_{x \in X}|\mu(x)|<\infty$, with norm given by this sum and order by $\nu \leqq \mu$ if $\nu(x) \leqq \mu(x)$ for all $x \in X$.

5. The second dual, $M$, and the imbedding of $C$. We denote the dual of $L$ by $M$. Like $C$ it is an $(M)$-space [11, Theorem 15$]$. We imbed $C$ in $M$ in 
the customary fashion $(f(\mu)=\mu(f)$ for all $\mu \in L)$. This imbedding of course preserves the norm. It also preserves the order; in fact

(5.1) For every $f \in C$ and $g \in C, f \vee g-i n-C=f \vee g-i n-M$ and $f \wedge g-i n$ $-C=f \wedge g-i n-M$.

Proof. It is enough to show that for every $f \in C, f^{+}-i n-C=f^{+}-i n-M$, that is (2.1), for every $\mu \in L_{+}, \mu\left(f^{+}-i n-C\right)=\sup _{0 \leq \nu \leq \mu} \nu(f)$. Consider $\mu \in L_{+}$; we can take $\|\mu\|=1$. Given $\epsilon>0$, let $Y=\{x \mid f(x) \geqq 0\}$ and $V=\{x \mid f(x)>$ $-\epsilon\}$. Then $Y$ is closed, $V$ open, and $Y \subset V$. Since $X$ is normal, there exists $g \in C$ such that $0 \leqq g \leqq 1, g(x)=1$ on $Y$, and $g(x)=0$ on the complement of $V$. Defining $g f$ by $(g f)(x)=g(x) f(x)$ for all $x \in X$, we have $g f \geqq\left(f^{+}-i n-C\right)-\epsilon 1$. Let $\nu$ be the element of $L$ defined by $\nu(h)=\mu(g h)$ for all $h \in C[2$, p. 43]. Then $0 \leqq \nu \leqq \mu$ and $\nu(f) \geqq \mu\left(f^{+}-i n-C\right)-\epsilon$.

As a result of the above theorem, we can henceforth write $f \vee g$ and $f \wedge g$ without ambiguity. In contradistinction, for a general subset $A$ of $C, \vee A$ and $\Lambda A$ will mean the supremum and infimum of $A$ in $M$. These differ in general from $\vee A-i n-C$ and $\Lambda A-i n-C$.

(5.2) The strong order unit 1 of $C$ is also the strong order unit of $M:$ for every $f \in M,\|f\| \leqq 1$ if and only if $|f| \leqq 1$.

Proof. The "if" of course is true in any Banach lattice. To show the "only if," suppose $\|f\| \leqq 1$. Then for every $\mu \in L_{+},|f|(\mu) \leqq\|f\|\|\mu\| \leqq\|\mu\|$ $=\mu(1)=1(\mu)$. Thus $|f| \leqq 1$.

Let $M_{0}=\left(L_{1}\right)^{\perp}$ and $M_{1}=\left(L_{0}\right)^{\perp}$ in $M$. From the general properties listed in $\S \S 1-3, M_{0}$ and $M_{1}$ are closed ideals in $M$, with $M_{1}=\left(M_{0}\right)^{\prime}$ and $M_{0}=\left(M_{1}\right)^{\prime}$, and $M$ is their topological direct sum. Moreover $M_{0}$ is the dual of $L_{0}$, hence from (4.6),

(5.3) $M_{0}$ can be identified with the Banach lattice of all bounded real functions on $X\left(\|f\|=\sup _{x \in X}|f(x)|, f \leqq g\right.$ if $f(x) \leqq g(x)$ for all $\left.x \in X\right)$.

For each $f \in M$, we will denote the components of $f$ in $M_{0}, M_{1}$ by $f_{0}, f_{1}$ respectively; and for each subset $A$ of $M$, we will denote the sets $\left\{f_{0} \mid f \in A\right\}$, $\left\{f_{1} \mid f \in A\right\}$ by $A_{0}, A_{1}$ respectively.

Now each $f \in C$ is completely determined by its values on $X$, hence the projection of $C$ onto $C_{0}$ is one-one. Moreover, from (5.3) and (5.1), it preserves the norm and order. We thus have

(5.4) $C$ is isometric and lattice-isomorphic with $C_{0}$.

We also have

(5.5) Let $A$ be a subset of $C$ and $f \in C$. Then $f=\bigvee A$ if and only if $f_{0}=\bigvee A_{0}$. (We recall that $\mathrm{V} A$ means $\mathrm{V} A-i n-M$ and $-M_{0}$ being a closed ideal - $\mathrm{V} A_{0}$ means $\mathrm{V} A_{0}-i n-M_{0}$; cf. remarks preceding (5.2).)

Proof. The "only if" follows from (1.4); we prove the "if". Since including the suprema of all finite subsets of $A$ does not change $\mathrm{V} A$ or $\mathrm{V} A_{0}$, and does not take us out of $C(5.1)$, we can assume $A$ is directed under $\leqq$. Suppose $f_{0}=\mathrm{V} A_{0}$. This means that the directed set of functions $A_{0}$ converges to $f_{0}$ at every $x \in X$, hence $A$ converges to $f$ at every $x \in X$. It follows from the Dini 
theorem that $A$ converges to $f$ uniformly on $X$, that is, under the norm. (3.4) now gives the desired conclusion.

It is important to keep clear the distinction between $M$ and $M_{0}$. In common practice $C$ is identified with $C_{0}$ and the (bounded) semi-continuous functions, Borel functions, and $\mu$-integrable functions $(\mu \in L)$ are all defined in $M_{0}$. In the present paper, $C$ is in general distinct from $C_{0}$ and does not lie in $M_{0}$, and the above classes of functions, as we will define them, will likewise in general not lie in $M_{0}$. The ones commonly worked with will be the projections of ours.

6. The semi-continuous elements of $M$. We will call $f \in M$ a lower-semicontinuous, or l.s.c., element if $f=\mathrm{V} A$ for some subset $A$ of $C$; we will call it an upper-semi-continuous, or u.s.c., element if $f=\Lambda A$ for some subset $A$ of $C$. The standard properties of vector lattices give us immediately

(6.1) If $f$ and $g$ are l.s.c., then so are $f+g, \lambda f$ ( $\lambda$ a positive real number), $f \vee g$, and $f \wedge g$.

(6.2) If $A$ is a set of l.s.c. elements and $f=\vee A$, then $f$ is l.s.c.

We also have

(6.3) If $f$ and $g$ are l.s.c., then $f \leqq g$ if and only if $f_{0} \leqq g_{0}$; in particular $f=g$ if and only if $f_{0}=g_{0}$.

Proof. The "only if" follows from (1.4). Now suppose $f_{0} \leqq g_{0}$. Let $f=\bigvee A$, $g=\mathrm{V} B$, where $A$ and $B$ are subsets of $C$; from (1.4) again, $f_{0}=\mathrm{V} A_{0}, g_{0}=\mathrm{V} B_{0}$. For a fixed $h \in A, h_{0} \leqq \bigvee_{k \in B} k_{0}$, hence $h_{0}=\bigvee_{k \in B}\left(k_{0} \wedge h_{0}\right)=\bigvee_{k \in B}(k \wedge h)_{0}$ (corollary to (1.4)). It follows from (5.5) that $h=\mathrm{V}_{k \in B}(k \wedge h) \leqq \mathrm{V}_{k \in B} k$. Since this holds for any $h \in A$, we have $\bigvee_{h \in A} h \leqq \mathrm{~V}_{k \in B} k$.

We will call the linear subspace of $M$ generated by the l.s.c. elements the space of semi-continuous elements, and denote it by $S$. It is easily shown that every element of $S$ can be written $f-g$, where $f$ and $g$ are l.s.c. elements. $S$ can also be defined as the linear subspace of $M$ generated by the u.s.c. elements; and every element of $S$ can be written as the difference of two u.s.c. elements.

(6.4) If $f$ and $g$ are elements of $S$, then so are $f \vee g$ and $f \wedge g$. In particular $f^{+}, f^{-}$, and $|f|$ are elements of $S$.

Proof. Let $f=f^{1}-f^{2}, g=g^{1}-g^{2}$, where $f^{1}, f^{2}, g^{1}, g^{2}$ are 1.s.c. Then $f \vee g$ $=\left(f^{1}-f^{2}\right) \bigvee\left(g^{1}-g^{2}\right)=\left(f^{1}+g^{2}\right) \bigvee\left(g^{1}+f^{2}\right)-\left(f^{2}+g^{2}\right)$. From (6.1) both terms in the last expression are l.s.c. The proof for $f \wedge g$ is the same.

(6.5) Given $f \in S, g \in S, f \leqq g$ if and only if $f_{0} \leqq g_{0}$; in particular, $f=g$ if and only if $f_{0}=g_{0}$.

Proof. Let $f=f^{1}-f^{2}, g=g^{1}-g^{2}$, where $f^{1}, f^{2}, g^{1}, g^{2}$ are 1.s.c. Suppose $f_{0} \leqq g_{0}$. Then $\left(f^{1}\right)_{0}-\left(f^{2}\right)_{0}=\left(f^{1}-f^{2}\right)_{0} \leqq\left(g^{1}-g^{2}\right)_{0}=\left(g^{1}\right)_{0}-\left(g^{2}\right)_{0} ;$ hence $\left(f^{1}+g^{2}\right)_{0}$ $=\left(f^{1}\right)_{0}+\left(g^{2}\right)_{0} \leqq\left(g^{1}\right)_{0}+\left(f^{2}\right)_{0}=\left(g^{1}+f^{2}\right)_{0}$. Applying (6.3) gives $f^{1}+g^{2} \leqq g^{1}+f^{2}$ and finally $f^{1}-f^{2} \leqq g^{1}-g^{2}$.

(6.6) For every $f \in S,\|f\|=\left\|f_{0}\right\|$.

Proof. Since $\left\|f_{0}\right\|=\left\|\left|f_{0}\right|\right\|=\left\||f|_{0}\right\|$ and $\|f\|=\||f|\|$, it is enough to prove 
the theorem for $f \geqq 0$. Now $0 \leqq f_{0} \leqq\left\|f_{0}\right\| 1_{0}$, hence from (6.5), $0 \leqq f \leqq\left\|f_{0}\right\| 1$, and thus $\|f\| \leqq\left\|f_{0}\right\|$. The converse inequality is trivial.

The last two theorems above give us that, as with $C$, the projection of $S$ on $S_{0}$ is one-one and preserves norm and order. Thus we have

(6.7) $S$ is isometric and lattice-isomorphic with $S_{0}$.

In general an element of $S$ does not lie in $M_{0}$. However

(6.8) If $f$ is the characteristic function in $M_{0}$ of a finite subset of $X$, then $f \in S$.

Proof. It is enough to prove this when the finite subset consists of a single point $x$. Let $A=\left\{g \mid g \in C_{+}, g(x) \geqq 1\right\}$ and $h=\bigwedge A$. Since $A$ is directed under $\geqq$, we have $h(\mu)=\inf _{g \in A} g(\mu)$ for all $\mu \in L_{+}$. This gives $h(x)=1$ and (from normality of $X) h(y)=0$ for all $y \neq x$; thus $h_{0}=f$. We show $h(\mu)=0$ for all $\mu \in\left(L_{1}\right)_{+}$, whence it will follow $h \in M_{0}$ and therefore $h=f$. Consider $\mu \in\left(L_{1}\right)_{+}$. $\mu \wedge x=0$, hence $\left[2\right.$, p. 36] given $\epsilon>0$, there exist $k \in C_{+}, l \in C_{+}$such that $k+l=1, \mu(k)<\epsilon$, and $l(x)<\epsilon$. We can assume $\epsilon<1 / 2$. Then $k(x)=1(x)-l(x)$ $>1-\epsilon$, whence $(1 / 1-\epsilon) k \in A$ and $\mu[(1 / 1-\epsilon) k]=(1 / 1-\epsilon) \mu(k)<2 \epsilon$. Thus $h(\mu)<2 \epsilon$. Since $\epsilon$ was arbitrary, we have $h(\mu)=0$.

(6.9) Every element of $S$ is an infimum of l.s.c. elements and a supremum of u.s.c. elements.

Proof. Consider $f=g-h$, where $g$ and $h$ are l.s.c. Then $h=\mathrm{V} A$, where $A \subset C$. It follows $f=\Lambda_{k \in A}(g-k)$. A similar argument holds for the second statement.

We give an example to show that the analogue of (5.5) is not true for $S$. Let $X$ be the real interval $0 \leqq x \leqq 1$. We note first that $L_{1}$ is not empty (it contains the Lebesgue measure, for example), hence that $M \neq M_{0}$ and therefore $1 \neq 1_{0}$. Now let $A$ be the set of all elements of $M_{0}$ which are characteristic functions of finite subsets of $X$. Then $A \subset S, 1 \in S$, and $1_{0}=\mathrm{V}_{A}=\mathrm{V} A_{0}$. However $1 \neq 1_{0}$, hence $1 \neq \vee A$. (We do have $1=\vee A-i n-S$, from (6.7)).

As we shall see later (8.5), the analogue of (5.5) is true under the additional condition that $A$ be countable.

Before leaving $S$ we establish a lemma which we will need later.

(6.10) If $A$ is a countable subset of $S$ and $f=\bigvee A$, then for every $\mu \in L_{+}$and $\epsilon>0$, there exists $g$ l.s.c. such that $g \geqq f$ and $g(\mu) \leqq f(\mu)+\epsilon$.

Proof. Let $A=\left\{f^{n}\right\}$. Since $f^{n}$ can be replaced by $\bigvee_{1}^{n} f^{i}(n=1,2, \cdots)$ without changing $f$, we can assume $f^{1} \leqq f^{2} \leqq \cdots$. We will obtain a bounded sequence $g^{1} \leqq g^{2} \leqq \cdots$ of 1 .s.c. elements such that $g^{n} \geqq f^{n}$ and $g^{n}(\mu) \leqq f^{n}(\mu)+\epsilon$ $(n=1,2, \cdots)$, and then $\bigvee_{n} g^{n}$ will be our required $g(2.2)$. Let $h^{1}=f^{1}$, $h^{n}=f^{n}-f^{n-1}(n=2,3, \cdots)$. Then for each $n, f^{n}=\sum_{1}^{n} h^{i}$. Since $h^{n} \in S$, we can write $h^{n}=h^{n, 1}-h^{n, 2}$, where $h^{n, 1}$ and $h^{n, 2}$ are 1.s.c. For each $n$, choose $k^{n} \in C$ to satisfy $k^{n} \leqq h^{n, 2}$ and $k^{n}(\mu) \geqq h^{n, 2}(\mu)-\epsilon / 2^{n} \quad(2.2)$. Let $l^{n}=h^{n, 1}-k^{n}$. Then $l^{n}$ is 1.s.c., $l^{n} \geqq h^{n}$, and $l^{n}(\mu) \leqq h^{n}(\mu)+\epsilon / 2^{n}$. Hence $\sum_{1}^{n} l^{i}$ is 1.s.c., $\sum_{1}^{n} l^{i} \geqq f^{n}$, and $\left(\sum_{1}^{n} l^{i}\right)(\mu) \leqq f^{n}(\mu)+\epsilon$. Now $f \leqq \lambda 1$ for some real number $\lambda$. Setting $g^{n}$ $=\left(\sum_{n}^{1} l^{i}\right) \wedge(\lambda 1)$, we obtain our required sequence.

7. The star elements of $M$. With each $f \in M$ we associate two elements 
$f_{*}, f^{*}$ defined by

$$
f_{*}=\underset{0 \in S, 0_{0} \leq f_{0}}{\bigvee} g, \quad f^{*}=\underset{0 \in S, 0_{0} \geq f_{0}}{\wedge} g .
$$

Since $f_{0} \leqq \lambda 1_{0}$ for some real number $\lambda$, the set defining $f_{*}$ is bounded above by $\lambda 1$ (6.7) and thus $f_{*}$ exists; and similarly for $f^{*}$.

We have immediately

(7.2) $\left(1^{\circ}\right) f \in S$ implies $f=f_{*}=f^{*}$.

$\left(2^{\circ}\right) f \in M, g \in S$ implies $(f+g)_{*}=f_{*}+g,(f+g)^{*}=f^{*}+g$.

$\left(3^{\circ}\right) f_{*} \leqq f^{*}$.

$\left(4^{\circ}\right) f_{0} \leqq g_{0}$ implies $f_{*} \leqq g_{*}, f^{*} \leqq g^{*}$; in particular, $f_{0}=g_{0}$ implies $f_{*}=g_{*}$, $f^{*}=g^{*}$.

$\left(5^{\circ}\right) f \leqq g$ implies $f_{*} \leqq g_{*}, f^{*} \leqq g^{*} ;$ in particular, $f \geqq 0$ implies $f_{*} \geqq 0, f^{*} \geqq 0$.

$\left(6^{\circ}\right)$ If $\lambda$ is a positive real number, $(\lambda f)_{*}=\lambda f_{*}$ and $(\lambda f)^{*}=\lambda f^{*}$.

$\left(7^{\circ}\right)(-f)_{*}=-f^{*}$.

Also, from (6.9),

$$
f_{*}=\underset{g \text { usc, } \theta_{0} \leq f_{0}}{\vee} g, \quad f^{*}=\wedge_{\theta \text { lsc, } \theta_{0} \geq f_{0}}^{\wedge} g .
$$

(7.4) $f_{*}$ and $f^{*}$ lie in the same coset of $M_{1}$ as $f$, that is, $\left(f_{*}\right)_{0}=\left(f^{*}\right)_{0}=f_{0}$.

Proof. Since we can always translate $f$ to $M_{+}$by adding a suitable multiple of 1 , it follows from $\left(2^{\circ}\right)$ above that we need only consider $f \geqq 0$. Let $A$ $=\left\{g \mid g \in S, g_{0} \leqq f_{0}\right\} ;$ then $\vee A=f_{*}$, hence $\vee A_{0}=\left(f_{*}\right)_{0}$. We show $\vee A_{0}=f_{0}$. It is trivial that $\bigvee A_{0} \leqq f_{0}$. To show the converse we note that if for each $x \in X$, $h_{x}$ is the element of $M_{0}$ which is the characteristic function of $x$, then $f(x) h_{x} \in A$ (6.8), hence $\in A_{0}$. It follows (5.3) that $\vee A_{0}=f_{0}$. That $\left(f^{*}\right)_{0}=f_{0}$ follows from $\left(7^{\circ}\right)$ above and the first part of the proof.

COROLlaRy. Every coset of $M_{1}$ contains exactly one lower-star element and upper-star element (they may coincide).

Straightforward computation gives

$$
f_{*}+g_{*} \leqq(f+g)_{*} \leqq f_{*}+g^{*} \leqq(f+g)^{*} \leqq f^{*}+g^{*},
$$

hence

$$
f_{*}-g^{*} \leqq(f-g)_{*} \leqq \begin{aligned}
& f^{*}-g^{*} \\
& f_{*}-g_{*}
\end{aligned}(f-g)^{*} \leqq f^{*}-g_{*} .
$$

These inequalities cannot be replaced by equalities, as the following example shows. Let $X$ be the real interval $0 \leqq x \leqq 1$. Let $Y$ and $Z$ be complementary subsets of $X$ such that the inner Lebesgue measure of each is 0 , and $f$ and $g$ be the elements of $M_{0}$ which are the characteristic functions of $Y$ and $Z$ respectively. Then $f+g=1_{0}$, hence $(f+g)_{*}=\left(1_{0}\right)_{*}=1\left(7.2\left(1^{\circ}\right)\right)$. We show $f_{*}+g_{*}<1$. To do this it is enough to show that $f_{*}(\mu)+g_{*}(\mu)<1(\mu)$ for one $\mu \in L_{+}$. Choose the Lebesgue measure for $\mu$. It is not hard to show that $f_{*}(\mu)$ and $g_{*}(\mu)$ are precisely the inner Lebesgue measures of $Y$ and $Z$ respectively. 
Since these are both 0 while $1(\mu)=1$, we are through. This establishes the necessity of the first inequality in the theorem; the same example can be used to show the necessity of the remaining ones.

Straightforward computation also gives

(7.6) For any bounded subset $A$ of $M$,

$$
\begin{aligned}
& \left(\bigwedge_{f \in A} f\right)_{*} \leqq \bigwedge_{f \in A} f_{*} \leqq \bigvee_{f \in A} f_{*} \leqq\left(\bigvee_{f \in A} f\right)_{*} . \\
& \left(\bigwedge_{f \in A} f\right)^{*} \leqq \bigwedge_{f \in A} f^{*} \leqq \bigvee_{f \in A} f^{*} \leqq\left(\bigvee_{f \in A} f\right)^{*}
\end{aligned}
$$

If $A$ is countable, the first inequality in $\left(1^{\circ}\right)$ and the last inequality in $\left(2^{\circ}\right)$ become equalities:

(7.7) For any countable bounded set $\left\{f^{n}\right\}$ in $M$,

$$
\left(\bigwedge_{n} f^{n}\right)_{*}=\bigwedge_{n}\left(f^{n}\right)_{*}, \quad\left(\bigvee_{n} f^{n}\right)^{*}=\bigvee_{n}\left(f^{n}\right)^{*}
$$

In particular, for any $f \in M$ and $g \in M$,

$$
(f \wedge g)_{*}=f_{*} \wedge g_{*}, \quad(f \vee g)^{*}=f^{*} \vee g^{*} .
$$

Proof. We prove the second equality in $\left(1^{\circ}\right)$; the first will follow by the duality relations within $M$ (cf. $\left(7^{\circ}\right)$ in $\left.(7.2)\right)$. From (7.6), we need only show $\left(\bigvee_{n} f^{n}\right)^{*} \leqq \bigvee_{n}\left(f^{n}\right)^{*}$. Let $f=\bigvee_{n}\left(f^{n}\right)^{*}$. Since $f_{0}=\bigvee_{n}\left(\left(f^{n}\right)^{*}\right)_{0}=\bigvee_{n}\left(f^{n}\right)_{0}$ $=\left(\bigvee_{n} f^{n}\right)_{0}$, it follows that $f^{*}=\left(\bigvee_{n} f^{n}\right)^{*}$. Hence what we need to show is that $f^{*} \leqq f$. Consider $\mu \in L_{+}$and $\epsilon>0$. From the definition (7.1), there exists a sequence $\left\{g^{n}\right\}$ of elements of $S$ such that $g^{n} \geqq\left(f^{n}\right)^{*}$ and $g^{n}(\mu) \leqq\left(f^{n}\right)^{*}(\mu)+\epsilon / 2^{n}$ $(n=1,2, \cdots)$. Let $h^{n}=\mathrm{V}_{1}^{n} g^{i}$. Then it is not hard to show that $h^{n}(\mu)$ $\leqq\left[V_{1}^{n}\left(f^{i}\right)^{*}\right](\mu)+\epsilon \leqq f(\mu)+\epsilon$. Applying (6.10), there exists $h \in S$ such that $h \geqq \bigvee_{n} h^{n} \geqq f$ and $h(\mu) \leqq\left(\bigvee_{n} h^{n}\right)(\mu)+\epsilon=\sup _{n} h^{n}(\mu)+\epsilon \leqq f(\mu)+\epsilon$. But $\epsilon$ was arbitrary and $\mu$ was any element of $L_{+}$, therefore $f^{*} \leqq f$.

In contradistinction to (7.7), the last inequality in $\left(1^{\circ}\right)$ and the first inequality in $\left(2^{\circ}\right)$ of $(7.6)$ are not replaceable by equalities even in the case of two elements. For, in the example following (7.5), $f \vee g=1_{0}$, hence $(f \vee g)_{*}$ * $=1$, but $f_{*} \bigvee g_{*} \neq 1$, since if $\mu$ is the Lebesgue measure, $\left(f_{*} \bigvee g_{*}\right)(\mu)=0$. However, see (8.13).

That (7.7) cannot be extended to uncountable sets is shown by the example following (6.9).

REMARK. The $f_{*}$ 's and $f^{*}$ 's can be defined independently of $M_{0}$, as follows. Let us call an ordered pair of disjoint sets $(A, B)$ of $S$ a Dedekind cut in $S$ if every element of $A$ is < every element of $B$ and the sets are maximal with respect to this property. Then we can define the $f_{*}$ 's as the suprema of lower members of Dedekind cuts and the $f^{* \prime} s$ as the infima of upper members. 
8. The universally integrable elements of $M$. If $f=f_{*}=f^{*}$, we will call $f$ universally integrable, and we will denote the set of all universally integrable elements of $M$ by $U$. $U_{0}$ consists precisely of the bounded functions on $X$ which are integrable, in the common sense of the term, with respect to every Radon measure on $X$.

(8.1) $U$ is a linear subspace.

Proof. If $f \in U, g \in U$, then from (7.5), $f+g=f_{*}+g_{*} \leqq(f+g)_{*} \leqq(f+g)^{*}$ $\leqq f^{*}+g^{*}=f+g$; thus $f+g \in U$. If $f \in U$ and $\lambda$ is a positive real number, then from (7.2), $(\lambda f)_{*}=\lambda f_{*}=\lambda f^{*}=(\lambda f)^{*}$; thus $\lambda f \in U$. If $f \in U$ and $\lambda$ is a negative real number, $(\lambda f)_{*}=-(-\lambda f)^{*}=-(-\lambda) f^{*}=\lambda f^{*}=\lambda f$, and similarly $(\lambda f)^{*}=\lambda f$; thus $(\lambda f)_{*}=(\lambda f)^{*}$, and $\lambda f \in U$.

(8.2) $U$ is $\sigma$-closed. Thus, in particular, it is a vector lattice.

Proof. Suppose $\left\{f^{n}\right\} \subset U$ and $f=\bigvee_{n} f^{n}$. Then $f=\bigvee_{n} f^{n}=\bigvee_{n}\left(f^{n}\right)_{*} \leqq\left(\bigvee_{n} f^{n}\right)_{*}$ $=f_{*} \leqq f^{*}=\left(\bigvee_{n} f^{n}\right)^{*}=\bigvee_{n}\left(f^{n}\right)^{*}=\bigvee_{n} f^{n}=f$, where for the crucial third-from-last equality we use (7.7).

(8.3) $U$ is isometric and lattice-isomorphic with $U_{0}$.

Proof. That the projection of $U$ on $U_{0}$ is one-one follows from the corollary of (7.4). The lattice-isomorphism follows from $\left(4^{\circ}\right)$ of $(7.2)$, and this in turn gives the isometry by the argument used in (6.6).

(8.4) $U$ is closed (hence complete) under the norm.

Proof. Suppose $f$ is a limit point of $U$. Then there is a sequence $\left\{f^{n}\right\}$ in $U$ such that $\left\|f^{n}-f\right\| \leqq 1 / n(n=1,2, \cdots)$. This says $\left|f^{n}-f\right| \leqq(1 / n) 1$ (5.2), whence $-(1 / n) 1 \leqq f^{n}-f \leqq(1 / n) 1$ or $f-(2 / n) 1 \leqq f^{n}-(1 / n) 1 \leqq f(n=1,2, \cdots)$. Thus $f=\mathrm{V}_{n}\left[f^{n}-(1 / n) 1\right]$. But $f^{n}-(1 / n) 1 \in U$ for all $n$, hence $f \in U$.

As we showed in $\S 6$, the analogue of (5.5) fails to be true in $S$, hence a fortiori in $U$. However

(8.5) Let $A$ be a countable subset of $U$ and $f \in U$. Then the following statements are equivalent:

$\left(1^{\circ}\right) f=\mathrm{V} A$;

(2 $\left.{ }^{\circ}\right)=\mathrm{V} A-i n-U$;

$\left(3^{\circ}\right) f_{0}=\mathrm{V} A_{0}$.

Proof. $\left(1^{\circ}\right)$ of course implies $\left(2^{\circ}\right)$ and $\left(3^{\circ}\right)$. That $\left(2^{\circ}\right)$ implies $\left(1^{\circ}\right)$ follows from (8.2). That $\left(3^{\circ}\right)$ implies $\left(2^{\circ}\right)$ follows from (8.3).

An easily verified property of $C$ is that if $f \in M$ is the supremum of a subset of $C$ and the infimum of a subset of $C$, then $f \in C$. We show $U$ has this property also.

(8.6) If $A$ and $B$ are subsets of $U$ and $f=\bigvee A=\Lambda B$, then $f \in U$.

Proof. From (7.6), $f^{*}=\left(\bigwedge_{h \in B} h\right)^{*} \leqq \bigwedge_{h \in B} h^{*}=\bigwedge_{h \in B} h=f=\bigvee_{o \in A} g=\bigvee_{g \in A} g_{*}$ $\leqq\left(\mathrm{V}_{g \in A} g\right)_{*}=f_{*}$.

We will call the smallest $\sigma$-closed linear subspace of $M$ containing $C$ the Baire subspace, and the smallest $\sigma$-closed linear subspace containing $S$ the Borel subspace [8]. We denote the former by $B a$ and the latter by $B o$. Since $U$ is $\sigma$-closed, we have immediately 


$$
B a \subset B o \subset U \text {. }
$$

Also

(8.8) Ba and Bo are each a vector lattice; each is isometric and latticeisomorphic with its projection on $M_{0}$; each is closed under the norm; and each has property (8.5).

The only statement here possibly requiring proof is the norm-closedness. But $1 \in B a$, hence the proof in (8.4) applies exactly.

REMARK. In some ways $U$ seems to be a more natural subspace of $M$ to work with than either $B o$ or $B a$. For example its definition is more natural in the sense that it does not require countability. Also it has the "semiDedekind" closedness described in (8.6). Most important of all, as we shall see in (9.6), $U$ consists precisely of the elements of $M$ which are limits under order-convergence of directed systems in $C$.

In the remainder of this $\S$ we apply $U$ to obtain additional properties of the star-elements.

(8.9) For every $f \in M, f_{*}=\bigvee_{g \in U, g_{0} \leqq f_{0}} g, f^{*}=\Lambda_{o \in U, g_{0} \geq f_{0}} g$.

This follows immediately from $\left(4^{\circ}\right)$ of $(7.2)$.

(8.10) $f \in M, g \in U$ implies $(f+g)_{*}=f_{*}+g,(f+g)^{*}=f^{*}+g$.

Proof. From (7.5), $(f+g)_{*} \leqq f_{*}+g^{*}=f_{*}+g=f_{*}+g_{*} \leqq(f+g)_{*}$. Similarly for the second equality.

(8.11) For every $f \in M,\left(f^{+}\right)^{*}=\left(f^{*}\right)^{+},\left(f^{+}\right)_{*}=\left(f_{*}\right)^{+},\left(f^{-}\right)^{*}=\left(f_{*}\right)^{-},\left(f^{-}\right)_{*}$ $=\left(f^{*}\right)^{-}$.

Proof. The first equality is contained in (7.7); we prove the second. We will assume for simplicity that $|f| \leqq 1$. From (7.6) we need only show $\left(f^{+}\right)$* $\leqq\left(f_{*}\right)^{+}$. Now $f_{*}=\bigvee_{h \in U, h \leqq f_{*}} h$, hence $\left(f_{*}\right)^{+}=\bigvee_{h \in U, h \leq f_{*}} h^{+}$. Also $\left(f^{+}\right)_{*}$

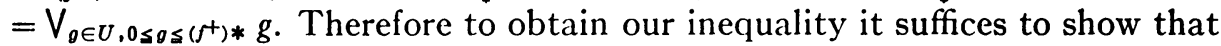
if $g \in U, 0 \leqq g \leqq\left(f^{+}\right)_{*}$, then $g=h^{+}$for some $h \in U, h \leqq f_{*}$. From (8.9) above and the isomorphism of $U$ with $U_{0}$, this reduces to proving that if $g \in U_{0}, 0 \leqq g$ $\leqq\left(f^{+}\right)_{0}=\left(f_{0}\right)^{+}$, then $g=h^{+}$for some $h \in U_{0}, h \leqq f_{0}$.

Let $k$ be the element of $M_{0}$ which is the characteristic function of the set $\{x \mid g(x)>0\}$. Since $k=\bigvee_{n=1}^{\infty}\left(1_{0} \wedge n g\right)$ and $1_{0} \wedge n g \in U_{0}$ for each $n$, we have $k \in U_{0}$ ( $U$ being $\sigma$-closed implies $U_{0} \sigma$-closed). Then $g-\left(1_{0}-k\right)$ is our required $h$. The remaining two equalities in the theorem follow from the first two and $\left(7^{\circ}\right)$ in (7.2).

As a corollary of (8.11) we have

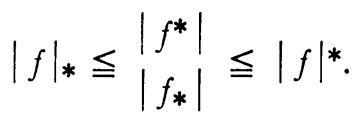

Proof. $\left|f_{*}\right|=\left(f_{*}\right)^{+}+\left(f_{*}\right)^{-}=\left(f^{+}\right)_{*}+\left(f^{-}\right)^{*}$ and $\left|f^{*}\right|=\left(f^{*}\right)^{+}+\left(f^{*}\right)^{-}=\left(f^{+}\right)^{*}$ $+\left(f^{-}\right)_{*}$. The required inequalities then follow from (7.5).

That the above inequalities are not replaceable by equalities can be shown by considering $-f$ in the example following (7.5). 
Finally from (8.10) and (8.11), we have

(8.13) If $f \in M$ and $g \in U$, then $(f \vee g)_{*}=f_{*} \vee g$ and $(f \wedge g)^{*}=f^{*} \wedge g$.

Proof. $f_{*} \vee g=g+\left(f_{*}-g\right)^{+}=g+\left[(f-g)_{*}\right]^{+}=g+\left[(f-g)^{+}\right]_{*}=\left[g+(f-g)^{+}\right]_{*}$ $=(f \vee g)_{*}$. The second equality follows from the first.

9. Order-convergence in $M$. Given a vector lattice $E$, the notation $\left\{a^{\alpha}\right\}$ will always denote a directed system, that is, the superscripts run through a partially ordered set $Q$, with the order denoted by $\prec$, say, such that for every pair $\alpha \in Q, \beta \in Q$, there exists $\gamma \in Q$ satisfying $\alpha<\gamma, \beta<\gamma$. In a complete lattice, if $\left\{a^{\alpha}\right\}$ is bounded, we define in the usual manner $\lim \inf a^{\alpha}=\mathrm{V}_{\alpha} \Lambda_{\beta}>_{\alpha} a^{\beta}$ and $\lim \sup a^{\alpha}=\bigwedge_{\alpha} \bigvee_{\beta}>_{\alpha} a^{\beta}$. If $\lim \inf a^{\alpha}=\lim \sup a^{\alpha}=a$, we will write $\lim a=a$ and say that $\left\{a^{\alpha}\right\}$ converges to $a$.

Since $M=\Omega(L)$, it shares with all $\Omega$ 's the following basic property, which, as can be seen, is Fatou's lemma in general form.

(9.1) Let $E$ be a vector lattice and $\left\{\phi^{\alpha}\right\}$ a bounded directed system in $\Omega(E)$. Then for every $a \in E_{+}, \quad\left(\lim \inf \phi^{\alpha}\right)(a) \leqq \lim \inf \phi^{\alpha}(a) \leqq \lim \sup \phi^{\alpha}(a)$ $\leqq\left(\lim \sup \phi^{\alpha}\right)(a)$.

Proof. Application of (both parts of) (2.2) gives $\left(\lim \inf \phi^{\alpha}\right)(a)$ $=\left(\bigvee_{\alpha} \bigwedge_{\beta}>_{\alpha} \phi^{\beta}\right)(a)=\sup _{\alpha}\left(\Lambda_{\beta}>_{\alpha} \phi^{\alpha}\right)(a) \leqq \sup _{\alpha} \inf _{\beta}>_{\alpha} \phi^{\beta}(a)=\lim \inf \phi^{\alpha}(a)$. This establishes the first inequality. A similar argument holds for the last.

Corollary. $\lim \phi^{\alpha}=\phi$ implies $\lim \phi^{\alpha}(a)=\phi(a)$ for every $a \in E$.

This follows from the theorem and the fact that every element of $E$ is the difference of two positive elements.

Turning to $M$ itself, we note first the following, which is limited to sequences because (7.7) holds only for countable sets.

(9.2) For any bounded sequence $\left\{f^{n}\right\}$ in $M$, we have

$\left(1^{\circ}\right) \lim \inf \left(f^{n}\right)_{*} \leqq\left(\lim \inf f^{n}\right)_{*} \leqq\left(\lim \inf f^{n}\right)^{*} \leqq \lim \inf \left(f^{n}\right)^{*}$;

$\left(2^{\circ}\right) \lim \sup \left(f^{n}\right)_{*} \leqq\left(\lim \sup f^{n}\right)_{*} \leqq\left(\lim \sup f^{n}\right)^{*} \leqq \lim \sup \left(f^{n}\right)^{*}$.

Proof. Lim inf $\left(f^{n}\right)_{*}=\bigvee_{n} \Lambda_{m \geq n}\left(f^{m}\right)_{*}=\bigvee_{n}\left(\Lambda_{m \geq n} f^{m}\right)_{*}$ (from (7.7)) $\leqq\left(\bigvee_{n} \Lambda_{m \geq n} f^{m}\right)_{*}($ from $(7.6))=\left(\lim \inf f^{n}\right)_{*}$. And similarly for the other inequalities

Combining (9.1) and (9.2), we obtain

(9.3) For any bounded sequence $\left\{f^{n}\right\}$ in $M$ and $\mu \in L_{+}$, we have

$$
\left[\lim \inf \left(f^{n}\right)_{*}\right](\mu) \leqq \begin{aligned}
& \liminf \left(f^{n}\right)_{*}(\mu) ; \\
& \left(\liminf f^{n}\right)_{*}(\mu) ;
\end{aligned}
$$

$$
\left(\lim \inf f^{n}\right)^{*}(\mu) \leqq\left[\lim \inf \left(f^{n}\right)^{*}\right](\mu) \leqq \lim \inf \left(f^{n}\right)^{*}(\mu) ;
$$$$
\left.\lim \sup \left(f^{n}\right)_{*}(\mu) \leqq\left[\lim \sup \left(f^{n}\right)_{*}\right](\mu) \leqq \lim \sup f^{n}\right)_{*}(\mu) ;
$$

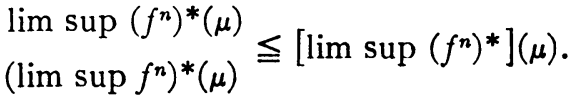


We proceed to examine $U$ with respect to convergence. The $\sigma$-closedness of $U$ gives immediately.

(9.4) If $\left\{f^{n}\right\}$ is a bounded sequence in $U$, then $\lim \inf f^{n} \in U$ and $\lim \sup f^{n} \in U$. In particular, $f=\lim f^{n}$ implies $f \in U$.

We also have

(9.5) If $\left\{f^{n}\right\}$ is a bounded sequence in $U$ and $f \in U$, the following statements are equivalent:

$\left(1^{\circ}\right) \lim f^{n}=f$;

$\left(2^{\circ}\right) \lim f^{n}(\mu)=f(\mu)$ for all $\mu \in L$;

$\left(3^{\circ}\right) \lim f^{n}(x)=f(x)$ for all $x \in X$, or, equivalently, $\lim \left(f^{n}\right)_{0}=f_{0}$.

Proof. That $\left(1^{\circ}\right)$ implies $\left(2^{\circ}\right)$ is contained in the Corollary to (9.1). That $\left(2^{\circ}\right)$ implies $\left(3^{\circ}\right)$ is of course trivial. That $\left(3^{\circ}\right)$ implies $\left(1^{\circ}\right)$ follows from the isomorphism theorem (8.3) and (9.4) above.

REMARK 1 . The statement that $\left(3^{\circ}\right)$ implies $\left(2^{\circ}\right)$ contains the essence of the Lebesgue bounded convergence theorem, at least, for norm-bounded sequences.

REMARK 2. That (9.5) does not hold for a general directed system is shown by the example following (6.9).

Finally, we prove that $U$ consists of the elements of $M$ which are limits under convergence of directed systems in $C$.

(9.6) Given $f \in M, f \in U$ if and only if $f=\lim f^{\alpha}$ for some directed system $\left\{f^{\alpha}\right\} \subset C$.

Proof. It is enough to establish the following two properties.

(I) If $\left\{f^{\alpha}\right\} \subset C, g=\lim \inf f^{\alpha}$, and $h=\lim \sup f^{\alpha}$, then $g \leqq g_{*}$ and $h \geqq h^{*}$.

(II) For any $f \in M$, there exists $\left\{f^{\alpha}\right\} \subset C$ such that $\lim \inf f^{\alpha}=f_{*}$ and $\lim \sup f^{\alpha}=f^{*}$.

We show first that the theorem follows from (I) and (II). If $f=\lim f^{\alpha}$, then from (I), $f \leqq f_{*} \leqq f^{*} \leqq f$; hence $f=f_{*}=f^{*}$. Conversely, if $f=f_{*}=f^{*}$, then from (II), there exists $\left\{f^{\alpha}\right\} \subset C$ such that $f=\lim \inf f^{\alpha}=\lim \sup f^{\alpha}$.

Now (I) follows directly from the fact that for each $\alpha, \Lambda_{\beta}>_{\alpha} f^{\beta} \in S$ and $\bigvee_{\beta}>{ }_{\alpha} f^{\beta} \in S$. We proceed to prove (II). Let $a=\{(g, h) \mid g$ u.s.c., $h$ 1.s.c., $\left.g_{0} \leqq f_{0} \leqq h_{0}\right\}$. Under the partial ordering $\left(g^{1}, h^{1}\right) \prec\left(g^{2}, h^{2}\right)$ if $g^{1} \leqq g^{2}, h^{1} \geqq h^{2}$, $Q$ is a directed set. By a standard property $[9$, p. 72], for each $\alpha=(g, h) \in Q$, there exists $f^{\alpha} \in C$ such that $g \leqq f^{x} \leqq h$. It follows from (7.3) (and (I)) that $\lim \inf f^{\alpha}=f_{*}$ and $\lim \sup f^{\alpha}=f^{*}$.

10. Weak topology. Given a vector lattice $E$, we will denote the weak topology on $\Omega(E)$ defined by $E$ by $w(\Omega, E)$. Three basic properties of $w(\Omega, E)$ are the following.

(10.1) Given $\left\{\phi^{\alpha}\right\} \subset \Omega$, if $\lim \phi^{\alpha}=\phi$, then $\lim \phi^{\alpha}=\phi$ under $w(\Omega, E)$.

(10.2) $\Omega_{+}$is complete under $w(\Omega, E)$.

(10.3) For every $\phi \in \Omega_{+}$, the "cube" $\{\psi|| \psi \mid \leqq \phi\}$ is compact under $w(\Omega, E)$.

(10.1) is the corollary to (9.1); while the arguments in [2, pp. 62,63] can clearly be applied to show (10.2) and (10.3) (also cf. [13]). 
Applying the above to $M$, we have

(10.4) An ideal $I$ in $M$ is closed if and only if it is closed under $w(M, L)$.

Proof. Suppose $I$ is closed. We remark first that the component $1_{I}$ of 1 in $I$ is the strong order unit for $I$, since $f \in I,\|f\| \leqq 1$ implies $|f| \leqq 1$ hence $|f| \leqq 1_{I}$. This says that the intersection of $I$ with the unit ball of $M$ is precisely the "cube" $\left\{f|| f \mid \leqq 1_{I}\right\}$, which from (10.3) above, is compact under $w(M, L)$. It follows from a theorem of Banach [3, Théorèmes 22, 23] that $I$ is closed under $w(M, L)$.

Suppose $I$ is closed under $w(M, L)$ and $f=\bigvee A, A \subset I$. Then $f=\lim f^{\alpha}$ for some directed system $\left\{f^{\alpha}\right\}$ in $I$. It follows from (10.1) that $f=\lim f^{\alpha}$ under $w(M, L)$, hence $f \in I$.

11. The Dieudonné topology. Given a vector lattice $E$, we consider the locally convex topology defined in $\Omega(E)$ by the polars of the bounded sets of $E$. Equivalently, if for each $a \in E_{+}$we define a semi-norm \|\|$_{a}$ on $\Omega$ by $\|\phi\|_{a}=|\phi|(a)$, the above topology is that defined by the family of all such semi-norms $[4 ; 5 ; 12 ; 13]$. Following Nakano, we will denote this topology by $|w|(\Omega, E)$. It is clearly finer than $w(\Omega, E)$ and has the nice property $[4 ; 13]$ :

(11.1) $\Omega$ is complete under $|w|(\Omega, E)$.

Each semi-norm in $|w|(\Omega, E)$ clearly has property (3.1), hence also (3.2), that is

(11.2) For every $a \in E_{+}$and elements $\phi, \psi, \rho$ of $\Omega,\|\phi \vee \rho-\psi \vee \rho\|_{a} \leqq\|\phi-\psi\|_{a}$ and $\|\phi \wedge \rho-\psi \wedge \rho\|_{a} \leqq\|\phi-\psi\|_{a}$. Thus the operations $\vee$ and $\wedge$ are uniformly continous under $|w|(\Omega, E)$.

In addition each semi-norm has the $(L)$-space property:

(11.3) For every $a \in E_{+}$and elements $\phi, \psi$ of $\Omega_{+},\|\phi+\psi\|_{a}=\|\phi\|_{a}+\|\psi\|_{a}$.

Consequently, the properties which followed from this in $\$ 4$ hold also here:

(11.4) If a set $A$ in $\Omega$ is directed under $\leqq$ and $\bigvee A=\phi$, then $\phi$ is a limit point of $A$ under $|w|(\Omega, E)$.

(11.5) An ideal in $\Omega$ is closed if and only if it is closed under $|w|(\Omega, E)$.

(11.6) If a subset $A$ of $\Omega$ is directed under $\leqq$ and bounded under $|w|(\Omega, E)$, then $\vee A$ exists.

This is proved in [5, Théorème 1$]$.

We can strengthen (10.1):

(11.7) Given $\left\{\phi^{\alpha}\right\} \subset \Omega$, if $\lim \phi^{\alpha}=\phi$, then $\lim \phi^{\alpha}=\phi$ under $|w|(\Omega, E)$.

Proof. For simplicity assume $\phi=0$. The statement $\lim \phi^{\alpha}=0$ is equivalent to the existence of a set $\left\{\psi^{\alpha}\right\}$, directed under $\geqq$ such that $\Lambda_{\alpha} \psi^{\alpha}=0$ and $\left|\phi^{\alpha}\right| \leqq \psi^{\alpha}$ for all $\alpha$. It follows from (the dual of) (11.4) that $\lim \psi^{\alpha}=0$ under $w \mid(\Omega, E)$, hence $\lim \phi^{\alpha}=0$ under $|w|(\Omega, E)$.

In $L,|w|(L, C)$ coincides with the norm topology, hence offers nothing new. In $M$, however, the situation is different. We remark first that from (10.4), (11.5), and (11.7) we have

(11.8) If $I$ is an ideal in $M$, the following statements are equivalent: 
$\left(1^{\circ}\right) I$ is closed under $w(M, L)$;

$\left(2^{\circ}\right) I$ is closed under $|w|(M, L)$;

$\left(3^{\circ}\right) I$ is closed under order-convergence;

$\left(4^{\circ}\right) I$ is closed.

We now proceed to prove the principal theorem of this section.

(11.9) $C$ is dense in $M$ under $|w|(M, L)$.

Proof. It is enough to show that for each $\mu \in L_{+},\|\mu\|=1, C$ is dense in $M$ under the semi-norm \|\|$_{\mu}$. The proof will be carried out by the now common technique of introducing an appropriate Hilbert space. We define an inner product in $C$ by $(f, g)=\mu(f g)$, where $f g$ is the usual multiplication: $(f g)(x)$ $=f(x) g(x)$. This inner product in turn gives us a Hilbert seminorm, $\|f\|_{2}$ $=[(f, f)]^{1 / 2}=\left[\mu\left(f^{2}\right)\right]^{1 / 2}$. (In this proof, unlike the remainder of the paper, a superscript is a power, not an index.) Since $f^{2}=|f|^{2}$ and $\|\mu\|=1$, we have immediately that $\|f\|_{2}=\||f|\|_{2}$ and $\|1\|_{2}=1$.

We note first that for every $f \in C$,

$$
\|f\|_{\mu} \leqq\|f\|_{2} \leqq\|f\| .
$$

For, $\|f\|_{\mu}=\mu(|f|)=\mu(1|f|)=(1,|f|) \leqq\|1\|_{2}\|f\|_{2}$ (Schwarz inequality) $=\|f\|_{2}$ $=\left[\mu\left(f^{2}\right)\right]^{1 / 2} \leqq\left[\|\mu\|\left\|f^{2}\right\|\right]^{1 / 2}=\left[\left\|f^{2}\right\|\right]^{1 / 2}=\left[\|f\|^{2}\right]^{1 / 2}=\|f\|$.

If we denote the unit balls of $C$ under \|\|$_{\mu},\|\|_{2},\|\|$ by $\Sigma_{\mu}(C), \Sigma_{2}(C)$, $\Sigma(C)$ respectively, (i) can be written $\Sigma(C) \subset \Sigma_{2}(C) \subset \Sigma_{\mu}(C)$. It follows $\Sigma_{\mu}(C)^{\circ}$ $C \Sigma_{2}(C)^{\circ} C \Sigma(C)^{\circ}$, where ${ }^{\circ}$ denotes the polar in $L$. Taking the polars of these latter sets in $M$, we obtain

$$
\Sigma(C)^{\circ \circ} C \Sigma_{2}(C)^{\circ \circ} C \Sigma_{\mu}(C)^{\circ \circ} \text {. }
$$

Now $\Sigma(C)^{\circ \circ}$ is the unit ball of $M$ under \|\| , hence spans $M$, and thus all three of these second polars span $M$ and therefore determine semi-norms on $M$. The first of these semi-norms is of course the norm \|\| . As for the second, the dual of $C$ under \|\|$_{2}$ is contained in $L$ (from (i)), hence $\Sigma_{2}(C)$ is closed in $C$ under $w(C, L)$, hence $\Sigma_{2}(C)^{\circ \circ} \cap C=\Sigma_{2}(C)$. It follows that the semi-norm determined on $M$ by $\Sigma_{2}(C)^{\circ \circ}$ is an extension to $M$ of \|\|$_{2}$, and we therefore denote it by the same symbol \|\|$_{2}$. Let $N$ be the null-space of \|\|$_{2}$ in $M$. Then \|\|$_{2}$ is a Hilbert norm on $C / N \cap C$, and it is easily seen that $M / N$ is contained in the second dual of $C / N \cap C$. It follows that $C / N \cap C$ is dense in $M / N$ under \|\|$_{2}$, hence $C$ is dense in $M$ under \|\|$_{2}$, hence from (ii), $C$ is dense in $M$ under the semi-norm defined by $\Sigma_{\mu}(C)^{\circ 0}$.

We complete the proof by showing that this last semi-norm is precisely \|\|$_{\mu}$. Let $A=\{\nu|\nu \in L,| \nu \mid \leqq \mu\}$. Then its polar $A^{\circ}$ in $M$ is the unit ball of $M$ under \|\|$_{\mu}$ and $\Sigma_{\mu}(C)=A^{\circ} \cap C$. It follows that $\Sigma_{\mu}(C)^{\circ}$ is the closure of $A$ under $w(L, C)$. But from (10.3), $A$ is compact under $w(L, C)$, hence $A$ $=\Sigma_{\mu}(C)^{\circ}$. We thus have $\Sigma_{\mu}(C)^{\circ \circ}=A^{\circ}$.

Corollary. $\Sigma(C)$ is dense in $\Sigma(M)$ under $|w|(M, L)(\Sigma(M)=$ the unit ball of $M$ under \|\|$)$. In fact $M_{+} \cap \Sigma(C)$ is dense in $M_{+} \cap \Sigma(M)$ under $|w|(M, L)$. 
Proof. Suppose $f \in M_{+} \cap \Sigma(M), \mu \in L_{+}$and $\epsilon>0$. From (11.9), $\|f-g\|_{\mu}<\epsilon$ for some $g \in C$. Then from (11.2), $\left\|f-g^{+} \wedge 1\right\|_{\mu}=\left\|f \wedge 1-g^{+} \wedge 1\right\|_{\mu} \leqq\left\|f-g^{+}\right\|_{\mu}$ $=\|f \vee 0-g \bigvee 0\|_{\mu} \leqq\|f-g\|_{\mu}<\epsilon$.

As we know from (10.3), the "cubes" in $L$ are compact under $w(L, C)$. (11.9) enables us to strengthen this considerably:

(11.10) For each $\mu \in L_{+}$, the "cube" $\{\nu|| \nu \mid \leqq \mu\}$ is compact under $w(L, M)$.

Proof. By the Dixmier-Grothendieck theorem [7], $C$ and $M$ determine the same weak topology on each such cube.

Denoting, as usual, the relatively strong topology of Mackey by $\tau(M, L)$ [6], (11.10) gives us

(11.11) $w(M, L) \subset|w|(M, L) \subset \tau(M, L)$ ( $\subset$ denotes "coarser than"). Thus a linear functional on $M$ is continuous under $|w|(M, L)$ if and only if it is an element of $L$.

12. Relation to integration theory. In this section we confine ourselves to a fixed $\mu \in L_{+},\|\mu\|=1$. We will denote by $L_{\mu}$ the closed ideal in $L$ generated by $\mu$, and by $M_{\mu}$ the (closed) ideal $\left(\left(L_{\mu}\right)^{\perp}\right)^{\prime}$ in $M$. It is easily shown that $M_{\mu}$ is a topological direct summand of $M$, and hence is the dual of $L_{\mu}$. As is known [4;5] $L_{\mu}$ can be identified with $\mathscr{L}^{1}(\mu)$ on $X$, and thus $M_{\mu}$ can be identified with $\mathfrak{L}^{\infty}(\mu)$. Note that $M_{\mu}$ does not in general lie in $M_{0}$; in fact if $\mu$ is nonatomic, $M_{\mu} \cap M_{0}=0$.

A development of integration theory in the context of $M$ (rather than $M_{0}$ ) would be a large task, and is not undertaken here. We content ourselves with giving some definitions and theorems showing how such a theory can be related to ordinary integration theory.

We first give the standard absolute continuity characterizations of $L_{\mu}$.

(12.1) Let $\Sigma(M)$ denote the unit ball of $M$ under \|\| . Then given $\nu \in L$, the following statements are equivalent:

$\left(1^{\circ}\right) \nu \in L_{\mu}$;

$\left(2^{\circ}\right) \nu$ is uniformly continuous on $\Sigma(M)$ under \|\|$_{\mu}$;

$\left(3^{\circ}\right) f(\nu)=0$ for every $f \in M$ satisfying $|f|(\mu)=0$.

Proof (After [5, p. 205]). Suppose $\nu$ is an element of the ideal $I$ generated by $\mu$, that is, $|\nu| \leqq \lambda \mu$ for some positive real number $\lambda$. Then for every $f \in M$, $g \in M,|\nu(f-g)|=|(f-g)(\nu)| \leqq|f-g|(|\nu|) \leqq \lambda|f-g|(\mu)=\lambda\|f-g\|_{\mu}$. Thus $\nu$ is uniformly continuous in $M$ under \|\|$_{\mu}$. Now $L_{\mu}$ is the closure of $I$ under the norm ((3.6) and (4.3)), therefore each element $\nu$ of $L_{\mu}$ is a uniform limit on $\Sigma(M)$ of a sequence of elements of $I$. From the above each element of the sequence is uniformly continuous on $\Sigma(M)$ under \|\|$_{\mu}$, hence $\nu$ is also. Thus $\left(1^{\circ}\right)$ implies $\left(2^{\circ}\right)$. That $\left(2^{\circ}\right)$ implies $\left(3^{\circ}\right)$ is obvious. Now suppose $\nu$ satisfies $\left(3^{\circ}\right)$. Then $\nu \in\left(L_{\mu}\right)^{\perp \perp}$. Since $L_{\mu}$ is closed under the norm, $L_{\mu}=\left(L_{\mu}\right)^{\perp \perp}$, and the proof is complete.

Corollary. $\left(L_{\mu}\right)^{\perp}$ in $M$ is the set $\{f|| f \mid(\mu)=0\}$.

Proof. $\left(L_{\mu}\right)^{\perp}$ is of course contained in this set. The statement: $\left(1^{\circ}\right)$ implies 
$\left(3^{\circ}\right)$ means that the set is contained in $\left(L_{\mu}\right)^{\perp}$.

We now turn to $M_{\mu}$. For each $f \in M$, we will denote the component of $f$ in $M_{\mu}$ by $f_{\mu}$; and for each subset $A$ of $M$, we will denote by $A_{\mu}$ the projection $\left\{f_{\mu} \mid f \in A\right\}$ of $A$ into $M_{\mu}$.

We note first that from the Corollary to $(12.1),\left(L_{\mu}\right)^{\perp}$ is precisely the nullspace of the semi-norm \|\|$_{\mu}$ and therefore \|\|$_{\mu}$ is a norm on $M_{\mu}$. Moreover, from $\left(2^{\circ}\right)$ of $(12.1)$, the topology defined by this norm coincides on $\Sigma\left(M_{\mu}\right)$ $\left(=M_{\mu} \cap \Sigma(M)\right.$ ) with $|w|(M, L)$ (they do not coincide on all of $M_{\mu}$ ).

The Corollary to (11.9) gives us

(12.2) $\Sigma\left(C_{\mu}\right)$ is dense in $\Sigma\left(M_{\mu}\right)$ under \|\|$_{\mu}$.

A stronger statement is:

(12.3) Given $f \in M_{\mu}, f=\lim f^{n}$ for some sequence $\left\{f^{n}\right\}$ in $C_{\mu}$.

Proof. We can assume $|f| \leqq 1_{\mu}$. From the above, there exists a sequence $\left\{f^{n}\right\}$ in $C$ such that $\left|f^{n}\right| \leqq 1_{\mu}$ for all $n$ and $\left\|f^{n}-f\right\|_{\mu} \leqq 1 / 2^{n}(n=1,2, \cdots)$. Let $g^{n}=\mathrm{V}_{m \geq n} f^{m}(n=1,2, \cdots)$. Then $\left\|g^{n}-f\right\|_{\mu}=\left\|\mathrm{V}_{m \geq n} f^{m}-f\right\|_{\mu}=\left\|\mathrm{V}_{m \geq n}\left(f^{m}-f\right)\right\|_{\mu}$ $\leqq\left\|\mathrm{V}_{m \geq n}\left|f^{m}-f\right|\right\|_{\mu}=\left\|\mathrm{V}_{p}\left(\mathrm{~V}_{m=n}^{p}\left|f^{n}-f\right|\right)\right\|_{\mu}=\sup _{p}\left\|\mathrm{~V}_{m=n}^{p}\left|f^{n}-f\right|\right\|_{\mu}(4.2)$

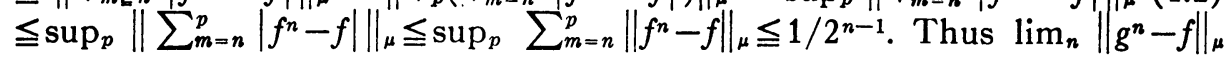
$=0$.

Now $\lim \sup f^{n}=\Lambda^{n} g^{n}$, hence (4.2) $\lim _{n}\left\|\lim \sup f^{n}-g^{n}\right\|_{\mu}=0$. Since $\left\|\lim \sup f^{n}-f\right\|_{\mu} \leqq\left\|\lim \sup f^{n}-g^{n}\right\|_{\mu}+\left\|g^{n}-f\right\|_{\mu}$, it follows that $\left\|\lim \sup f^{n}-f\right\|_{\mu}$ $=0$. But \|\|$_{\mu}$ is a norm on $M_{\mu}$, hence $\lim \sup f^{n}=f$. Repeating the entire argument gives us $\lim \inf f^{n}=f$, which completes the proof.

(12.3) in turn gives us

$$
M_{\mu}=U_{\mu}=(B o)_{\mu}=(B a)_{\mu} .
$$

Proof. This says that $B a, B o$, and $U$ all project onto $M_{\mu}$. We need of course only show this for $B a$. Consider $f \in M_{\mu}$, and for simplicity suppose $|f| \leqq 1$. By (12.3) $f=\lim f^{n}$ for some sequence $\left\{f^{n}\right\}$ in $C_{\mu}$. Since this convergence still holds if we replace each $f^{n}$ by $\left(f^{n} \wedge 1\right) \bigvee(-1)$, we can also assume $\left|f^{n}\right| \leqq 1$ for all $n$. Now, for each $n$, choose $g^{n} \in C$ having $\left(g^{n}\right)_{\mu}=f^{n}$, and again we can assume $\left|g^{n}\right| \leqq 1$. Let $g=\lim \sup g^{n} \in B a$. It follows easily that $g_{\mu}=\lim \sup f^{n}=f$.

We now come specifically to the relationship between $M_{\mu}$ and integration theory. In the following, $\left(L_{\mu}\right)^{\perp}$ will be denoted by its equivalent symbol $\left(M_{\mu}\right)^{\prime}$.

(12.5) The following two ideals in $M$ are identical:

(a) the ideal generated by $\left(M_{\mu}\right)^{\prime} \cap U$;

(b) the ideal generated by the f*'s lying in $\left(\left(M_{\mu}\right)^{\prime}\right)_{+}$.

$W e$ will denote this ideal by $N$, and call its elements $\mu$-negligible.

Proof. Suppose $f \geqq 0$ is an element of the first ideal. Then $f \leqq g$ for some $g \in\left(M_{\mu}\right)^{\prime} \cap U$. Since $g=g^{*}$, it follows $f$ is an element of the second ideal. Conversely, suppose $f \geqq 0$ lies in the second ideal. Then $f \leqq g$ for some $g=g^{*} \in\left(M_{\mu}\right)^{\prime}$. Now $g=\Lambda_{h \in S, h \geq g} h$, hence $0=g(\mu)=\inf _{h \in S, h \geq g} h(\mu)$. It follows there exists a sequence $\left\{h^{n}\right\}$ such that $h^{n} \in S, h^{n} \geqq g, h^{n}(\mu) \leqq 1 / n(n=1,2, \cdots)$. Setting $h=\Lambda_{n} h^{n}$, we have $h \in\left(M_{\mu}\right)^{\prime} \cap U$ and $f \leqq h$; thus $f$ is an element of the first 
ideal. We have shown the positive cones of the two ideals are identical; it follows the ideals are also.

(12.6) $N$ is $\sigma$-closed.

Proof. Since $\left(M_{\mu}\right)^{\prime}$ and $U$ are both $\sigma$-closed, $\left(M_{\mu}\right)^{\prime} \cap U$ is also. Consider a countable subset $\left\{f^{n}\right\}$ of $N_{+}$, which is bounded above by 1 , say. Now for each $n, f^{n} \leqq g^{n}$ for some $g^{n} \in\left(M_{\mu}\right)^{\prime} \cap U$. Since $g^{n} \wedge 1 \in\left(M_{\mu}\right)^{\prime} \cap U$ also, we can assume for simplicity that $g^{n} \leqq 1(n=1,2, \cdots)$. We thus have $\bigvee_{n} f^{n} \leqq V_{n} g^{n}$ $\in\left(M_{\mu}\right)^{\prime} \cap U$, hence $\mathrm{V}_{n} f^{n} \in N$.

(12.7) Given $f \in M$, the following statements are equivalent:

$\left(1^{\circ}\right) f_{0} \in N$;

$\left(2^{\circ}\right) f_{*} \in N$;

$\left(3^{\circ}\right) f^{*} \in N$

$\left(4^{\circ}\right)$ Some element of the coset $f+M_{1}$ lies in $N$.

Proof. We need only show that $\left(4^{\circ}\right)$ implies $\left(1^{\circ}\right),\left(2^{\circ}\right)$, and $\left(3^{\circ}\right)$. For simplicity of notation, assume $f \in N$. Suppose first that $f \geqq 0$. Then $f \leqq g$ $\in\left(M_{\mu}\right)^{\prime} \cap U$. Applying (7.2), we have $0 \leqq f_{0} \leqq f_{*} \leqq f^{*} \leqq g^{*}=g$, whence $f_{0}, f_{*}, f^{*}$ all lie in $N$. For a general $f \in N$, we have $f^{+} \in N$ and $f^{-} \in N$, hence by the argument just used, $\left(f^{+}\right)_{0},\left(f^{+}\right)_{*},\left(f^{+}\right)^{*},\left(f^{-}\right)_{0},\left(f^{-}\right)_{*},\left(f^{-}\right)^{*}$ all lie in $N$. It follows from the corollary to (1.4) and (8.11) that $f_{0}, f_{*}$, and $f^{*}$ lie in $N$.

Corollary. $N_{0}=N \cap M_{0}$.

We will denote by $\mathfrak{M}$ the linear subspace $U+N$, and we will call its elements $\mu$-integrable. From (12.4) each element of $U$ differs from an element of $B a$ by an element of $\left(M_{\mu}\right)^{\prime}$, hence by an element of $N$. It follows $\mathfrak{T}$ $=B a+N$.

REMARK. In the last statement we used the fact, true by definition, that an element of $U$ which lies in $\left(M_{\mu}\right)^{\prime}$ lies in $N$. We note for later reference that the same is true for any element of $\mathfrak{T}$, as is easily verified.

(12.8) Given $f \in M$, the following statements are equivalent:

(1 $\left.{ }^{\circ}\right) f_{0} \in \mathscr{M}_{0}$;

$\left(2^{\circ}\right) f_{*} \in \mathscr{M}$;

$\left(3^{\circ}\right) f^{*} \in \mathscr{T}$;

$\left(4^{\circ}\right) f^{*}-f_{*} \in N$;

(5) $f^{*}-f_{*} \in\left(M M_{\mu}\right)^{\prime}$

$\left(6^{\circ}\right) f^{*}(\mu)=f_{*}(\mu)$.

And if any (hence all) of these conditions hold, $\mathfrak{T} \cap\left(f+M_{1}\right)$ lies in a single coset of $N$.

Proof. $\left(1^{\circ}\right)$ implies $\left(2^{\circ}\right)$ and $\left(3^{\circ}\right):$ We can suppose $f \in \Re$. This says $f=g+h, g \in U, h \in N$. From (12.7), $h_{*} \in N, h^{*} \in N$, hence (8.10) $f_{*}=g+h_{*} \in \mathscr{M}$ and $f^{*}=g+h^{*} \in \mathfrak{M}$. That $\left(2^{\circ}\right)$ and $\left(3^{\circ}\right)$ each imply $\left(1^{\circ}\right)$ is trivial. $\left(1^{\circ}\right)$ implies $\left(4^{\circ}\right)$ : In the notation just used, $f^{*}-f_{*}=h^{*}-h_{*} \in N$. $\left(4^{\circ}\right)$ of course implies $\left(5^{\circ}\right)$, and $\left(5^{\circ}\right)$ implies $\left(6^{\circ}\right)$. We complete the equivalence by showing $\left(6^{\circ}\right)$ implies $\left(3^{\circ}\right)$. By the same argument as was used in (12.5), there exist $g \in U$, 
$h \in U$ such that $g \leqq f_{*} \leqq f^{*} \leqq h$ and $g(\mu)=f_{*}(\mu)=f^{*}(\mu)=h(\mu)$. Then from the Corollary to (12.1), $h-g \in\left(M_{\mu}\right)^{\prime} \cap U$. Now $0 \leqq f^{*}-g \leqq h-g$, hence $f^{*}-g \in N$. Writing $f^{*}=g+\left(f^{*}-g\right)$, we have $f^{*} \in U+N$. To prove the concluding statement we need only show that if $f \in \mathfrak{M}, f-f_{*} \in N$. In the notation used at the beginning of the proof, $f-f_{*}=h-h_{*} \in N$.

(12.9) $\mathfrak{T}$ is $\sigma$-closed.

Proof.

LeMma 1. $f \in \mathscr{T}$ implies $f^{+} \in \mathscr{T}$.

We note first that $\left(f_{*}\right)^{+} \in$ IT. For, from $(8.11) 0 \leqq\left(f^{+}\right)^{*}-\left(f^{+}\right)_{*}=\left(f^{*}\right)^{+}$ $-\left(f_{*}\right)^{+} \leqq\left(f^{*}-f_{*}\right)^{+}=f^{*}-f_{*}$. From (12.8) the last expression lies in $N$, therefore also the first, and from (12.8) again, $\left(f^{+}\right)_{*} \in \mathfrak{T}$. Turning to $f^{+},-\left(f_{*}-f\right)^{+}$ $\leqq f^{+}-\left(f_{*}\right)^{+} \leqq\left(f-f_{*}\right)^{+}$. Since $f-f_{*} \in N(12.8)$, we have $-\left(f_{*}-f\right)^{+}$and $\left(f-f_{*}\right)^{+}$ both in $N$. Thus $f^{+}-\left(f_{*}\right)^{+}$lies between two elements of $N$, hence is itself an element of $N$. Writing $f^{+}=\left(f_{*}\right)^{+}+\left(f^{+}-\left(f_{*}\right)^{+}\right)$, we have $f^{+} \in \mathfrak{T}$.

Lemma 2. Given $f \in \mathfrak{M}, 0 \leqq f \leqq 1$, we can write $f=g+h, g \in U, h \in N$ such that $0 \leqq g \leqq 1$. It follows that if $|f| \leqq 1$, we can write $f=g+h, g \in U, h \in N$ such that $|g| \leqq 1$.

Since $f \in \mathscr{T}, f=g^{1}+h^{1}, g^{1} \in U, h^{1} \in N$. We show $\left(g^{1}\right)^{+} \bigwedge 1$ is the required $g$. Writing $f=\left(g^{1}\right)^{+} \wedge 1+\left(h^{1}+g^{1}-\left(g^{1}\right)^{+} \wedge 1\right)$ we see we need only show $g^{1}-\left(g^{1}\right)^{+}$ $\wedge 1 \in\left(M_{\mu}\right)^{\prime} . \quad\left(g^{1}\right)_{\mu}=f_{\mu}$, hence $\left(\left(g^{1}\right)^{+} \wedge 1\right)_{\mu}=\left(\left(g^{1}\right)^{+}\right)_{\mu} \wedge 1_{\mu}=\left(\left(g^{1}\right)_{\mu}\right)^{+} \wedge 1_{\mu}=\left(f_{\mu}\right)^{+}$ $\wedge 1_{\mu}=f_{\mu} \wedge 1_{\mu}=f_{\mu}$. It follows $\left(g^{1}-\left(g^{1}\right)+\wedge 1\right)_{\mu}=f_{\mu}-f_{\mu}=0$. The last part of the lemma follows easily by applying the first part to $f^{+}$and $f^{-}$separately.

We proceed to prove the theorem. From Lemma $1, \mathfrak{T}$ is a vector lattice, hence it suffices to show that if $\left\{f^{n}\right\} \subset \mathfrak{M}_{+}, f^{1} \leqq f^{2} \leqq \cdots \leqq 1$, then $\bigvee_{n} f^{n} \in \mathscr{M}$. By Lemma 2, we have for each $n, f^{n}=g^{n}+h^{n}, g^{n} \in U, h^{n} \in N$, where $0 \leqq g^{n} \leqq 1$. We show first that we can also assume $g^{1} \leqq g^{2} \leqq \cdots$. Writing $f^{2}=g^{2} \bigvee g^{1}$ $+\left(h^{2}+g^{2}-g^{2} \bigvee g^{1}\right)$, we have $\left(g^{2}-g^{2} \bigvee g^{1}\right)_{\mu}=\left(g^{2}\right)_{\mu}-\left(g^{2}\right)_{\mu} \bigvee\left(g^{1}\right)_{\mu}=\left(f^{2}\right)_{\mu}-\left(f^{2}\right)_{\mu}$ $\bigvee\left(f^{1}\right)_{\mu}=\left(f^{2}\right)_{\mu}-\left(f^{2}\right)_{\mu}=0$, and hence $h^{2}+g^{2}-g^{2} \bigvee g^{1} \in N$. Thus we can replace $g^{2}$ by $g^{2} \vee g^{1}$ if necessary; and the same argument applies to all the $f^{n}$ by induction.

Now let $f=\bigvee_{n} f^{n}$ and $g=\bigvee_{n} g^{n}$; since $\left\{f^{n}\right\}$ and $\left\{g^{n}\right\}$ are ascending sequences, $f=\lim f^{n}$ and $g=\lim g^{n}$. It follows from $h^{n}=f^{n}-g^{n}$ that $\left\{h^{n}\right\}$ converges and $\lim h^{n}=\lim f^{n}-\lim g^{n}=f-g$. Denoting $\lim h^{n}$ by $h$, we have $f=g+h$. Now $U$ and $N$ are $\sigma$-closed, hence $g \in U, h \in N$. This gives $f \in \Re$.

Our final theorem gives the equivalents of convergence almost everywhere (for bounded sequences).

(12.10) Let $\left\{f^{n}\right\}$ be a bounded sequence in $\mathfrak{T}$. Then the following statements are equivalent:

$\left(1^{\circ}\right)\left\{\left(f^{n}\right)_{\mu}\right\}$ converges;

(2) $\lim \sup f^{n}-\lim \inf f^{n} \in\left(M_{\mu}\right)^{\prime}$;

$\left(3^{\circ}\right) \lim \sup f^{n}-\lim \inf f^{n} \in N$; 
$\left(4^{\circ}\right) \lim \sup \left(f^{n}\right)_{0}-\lim \inf \left(f^{n}\right)_{0} \in N$.

Proof. That $\left(1^{\circ}\right)$ and $\left(2^{\circ}\right)$ are equivalent is obvious. That $\left(2^{\circ}\right)$ and $\left(3^{\circ}\right)$ are equivalent follows from (12.9) and the Remark preceding (12.8). Since $\left(3^{\circ}\right)$ of course implies $\left(4^{\circ}\right)$, we need only prove that $\left(4^{\circ}\right)$ implies $\left(3^{\circ}\right)$. For each $n, f^{n}=g^{n}+h^{n}, g^{n} \in U, h^{n} \in N$, and from Lemma 2 of (12.9) we can assume the sequences $\left\{g^{n}\right\}$ and $\left\{h^{n}\right\}$ are bounded. Now $\lim \inf g^{n}+\lim \inf h^{n}$ $\leqq \lim$ inf $\left(g^{n}+h^{n}\right) \leqq \lim$ sup $\left(g^{n}+h^{n}\right) \leqq \lim$ sup $g^{n}+\lim$ sup $h^{n} ;$ hence 0 $\leqq \lim \sup f^{n}-\lim \inf f^{n} \leqq\left(\lim \sup g^{n}-\lim \inf g^{n}\right)+\left(\lim \sup h^{n}-\lim \inf h^{n}\right)$. To obtain the desired conclusion it suffices to show lim sup $g^{n}-\lim$ inf $g^{n} \in N$. (lim sup $g^{n}-\lim$ inf $\left.g^{n}\right)_{0}=\lim \sup \left(g^{n}\right)_{0}-\lim \inf \left(g^{n}\right)_{0}$. This last expression lies in $N$, as can be verified by writing $\left(g^{n}\right)_{0}=\left(f^{n}\right)_{0}-\left(h^{n}\right)_{0}$ and applying the same kind of inequalities we have just run through. Further, lim sup $g^{n}$ $-\lim \inf g^{n}=\left(\lim \sup g^{n}-\lim \inf g^{n}\right)_{*}$, since it lies in $U$; hence applying (12.7), we have lim sup $g^{n}-\lim \inf g^{n} \in N$.

The implication: $\left(4^{\circ}\right)$ implies $\left(1^{\circ}\right)$, together with the corollary to (9.1) gives us the

Corollary (Lebesgue bounded convergence theorem). Given a bounded sequence $\left\{f^{n}\right\}$ in $M$, if $\lim \sup \left(f^{n}\right)_{0}-\lim \inf \left(f^{n}\right)_{0} \in N$, then $\left\{f^{n}(\mu)\right\}$ converges.

\section{BiBLIOGRAPHY}

1. G. Birkhoff, Lattice theory, 1948.

2. N. Bourbaki, Integration, Chapters I-IV, 1952 (vol. XIII of Eléments de mathematique).

3. J. Dieudonné, La dualité dans les espaces vectoriels topologiques, Ann. Ecole Norm. vol. 59 (1942) pp. 108-139.

4. - Sur le théoréme de Lebesgue-Nikodym, Ann. of Math. vol. 42 (1941) pp. 547-556.

5. - Sur le théoréme de Lebesgue-Nikodym (II), Bull. Soc. Math. France vol. 72 (1944) pp. 193-240.

6. J. Dieudonné and L. Schwartz, La dualité dans les espaces $(F)$ et $(L F)$, Ann. de l'Institut Fourier vol. 1 (1949) pp. 61-101.

7. A. Grothendieck, Sur la completion du dual d'un espaces vectoriel localement convexe, C. R. Acad. Sci. Paris vol. 230 (1950) pp. 605-606.

8. P. R. Halmos, Measure theory, 1950.

9. N. Bourbaki, Topologie générale, Chapter IX, 1948.

10. S. Kakutani, Concrete representation of abstract $(L)$-spaces and the mean ergodic theorem, Ann. of Math. vol. 42 (1941) pp. 523-537.

11. - Concrete representation of abstract (M)-spaces, Ann. of Math. vol. 42 (1941) pp. 994-1024.

12. H. Nakano, Linear topologies on semi-ordered linear spaces, Journal of the Faculty of Science. Hokkaido Imperial University vol. 12 (1953) pp. 87-105.

13. G. T. Roberts, Topologies in vector lattices, Proc. Cambridge Phil. Soc. vol. 48 (1952) pp. 533-547.

Wayne State University, Detroit, Mich. 